\title{
Influence of the Nursing Effect on the Antiinflammatory Effect of Atorvastatin in Acute Cerebral Infarction
}

YI XU1', YIYING WANG ${ }^{2}$ AND JIANRONG WANG ${ }^{3 *}$

${ }^{1}$ College of Medicine (School of Nursing), Chengdu University, Chengdu 610106, ${ }^{2}$ School of Basic Medicine Sciences, Chongqing Medical University, Chongqing 400016, ${ }^{3}$ Neurology Department, Chengdu Fifth People's Hospital, Chengdu 610051, China 
To observe the antiinflammatory effect and nursing effect of atorvastatin on acute cerebral infarction, 180 patients with acute cerebral infarction who were treated in the Chengdu Fifth People's Hospital were selected as research subjects. They were divided into the control group (90) which received the routine treatment and the research group which was given atorvastatin treatment (90 cases). In addition, the patients in the research group were provided with a comprehensive nursing intervention program, while the control group was given routine nursing mode. The therapeutic effects and nursing effects of the two groups were compared. By comparing the serum lipid-related indices of the two groups, it was found that the patients in the research group had a significant advantage over the control group. Through observing and comparing the levels of markers of immune inflammation, the improvement in the serum tumor necrosis factor level, IL-6 and intercellular adhesion molecule 1 concentrations in the research group was more significant, $\mathbf{P}<\mathbf{0 . 0 5}$. In addition, the comprehensive life quality evaluation score and FMA exercise ability score of the research group showed significant advantages, $P<0.05$. For patients with acute cerebral infarction, atorvastatin treatment can produce good antiinflammatory effect and the treatment effect can be significantly improved after adopting scientific nursing measures.

Key words: Cerebral infarction, acute phase, atorvastatin, antiinflammatory effect, nursing intervention, efficacy observation

Cerebral infarction is also called ischemic stroke or stroke in Chinese medicine. Due to blood supply disorder in local brain tissue areas, brain tissue ischemic and hypoxic lesions and necrosis can result, which thus leads to clinical manifestations of neurological deficit, called cerebral infarction. According to the difference in pathogenesis, cerebral infarction can be divided into cerebral thrombosis, cerebral embolism and lacunar cerebral infarction ${ }^{[1-3]}$. Among these, cerebral thrombosis is the most common type of cerebral infarction, accounting for about $60 \%$ of all cerebral infarctions. Therefore, the commonly called cerebral infarction actually refers to cerebral thrombosis. Acute cerebral infarction refers to brain necrosis caused by sudden interruption of cerebral blood supply as shown in fig. 1. Usually, the acute cerebral infarction is a result of atherosclerosis and thrombosis in cerebral arteries, making the lumen narrow or even blocked, leading to the focal acute cerebral insufficiency. In addition, abnormal objects such as solid, liquid, and gas enter the cerebral artery or the neck of the artery through circulation result in blocking of blood flow or sudden reduction in blood flow leading to brain tissue softening and necrosis in the corresponding area supplied by the artery and thus causing acute cerebral infarction ${ }^{[4-12]}$. The acute cerebral infarction poses a serious threat to patient's life and it is essential to take active and effective drug treatment and scientific nursing intervention programs. The following is an exploration of the antiinflammatory effect and nursing effect of atorvastatin on acute cerebral infarction.

In this study, 180 patients who had been treated in Chengdu Fifth People's hospital for acute cerebral infarction (fig. 2) from January 2015 to June 2018 were selected as research subjects. The inclusion criteria were all those patients who met the diagnostic criteria for cerebrovascular diseases formulated by the $4^{\text {th }}$ national conference on cerebrovascular diseases in 1995. The selected patients were randomly divided into the research group (90) and the control group (90). In the research group, there were 50 males and 40 females, the oldest was of $72 \mathrm{y}$, the youngest was of $36 \mathrm{y}$, and the average age was $48.6 \pm 3.5 \mathrm{y}$. In the control group, there were 55 males and 35 females, the oldest was of $72 \mathrm{y}$, the youngest was of $38 \mathrm{y}$ and the average was $47.9 \pm 3.0 \mathrm{y}$. There were no significant differences in the general data between the two groups before treatment. After admission, the patients in the control group were given routine treatment that included dehydration to reduce intracranial pressure, maintenance of water and electrolyte balance and antiinfective therapy. In addition, $20 \mathrm{mg}$ of edaravone was dissolved in $250 \mathrm{ml}$ of sodium chloride $(0.9 \%)$ solution and administered by intravenous infusion twice a day. Moreover, these patients were given general routine nursing support, such as environmental nursing, drug guidance and monitoring of vital signs. The treatment regimen was continued for $1 \mathrm{mo}$. In similar lines of the therapy given 


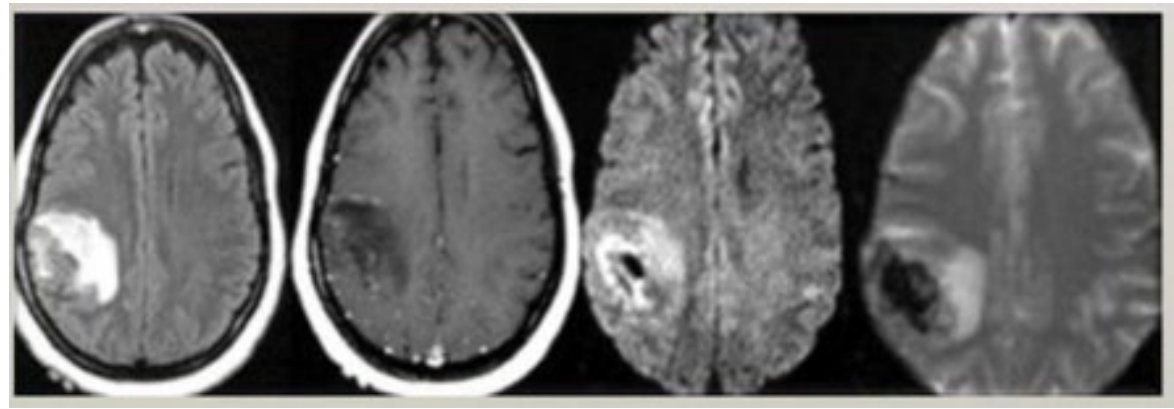

Fig. 1: Image of acute cerebral infarction
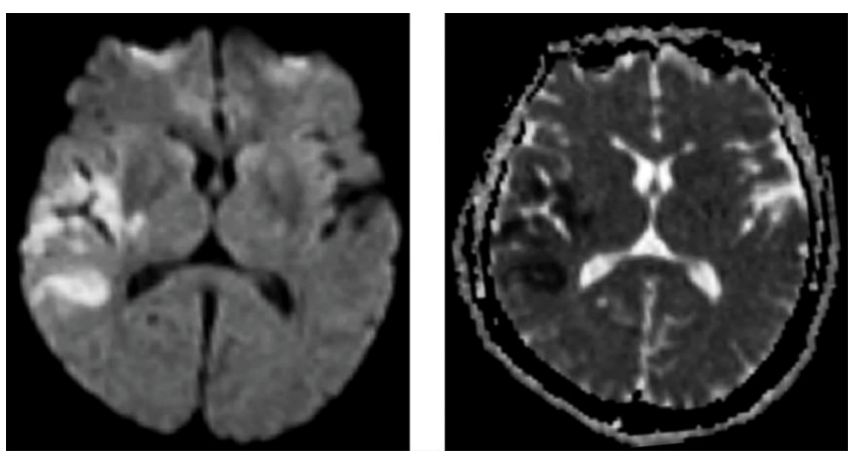

Fig. 2: Examination chart of a patient with acute cerebral infarction

to the control group, the patients in the research group were administered orally with $20 \mathrm{mg}$ of atorvastatin, once a day at bedtime for $1 \mathrm{mo}$. In addition, a comprehensive nursing intervention measure was implemented for these patients, which included psychological guidance, environmental nursing, health promotion education, diet nursing and so on.

Serum total cholesterol (TC), triglyceride (TG), lowdensity lipoprotein cholesterol (LDL-C) and highdensity lipoprotein cholesterol (HDL-C) were measured before and after treatment. Serum inflammatory markers were detected as well, including C-reactive protein (CRP), tumor necrosis factor $\alpha$ (TNF- $\alpha$ ), interleukin 1 (IL-1), interleukin 6 (IL-6), interleukin 10 (IL-10), E-selectin, P-selectin, vascular cell adhesion molecule-1 (VCAM-1) and intercellular adhesion molecule-1 (ICAM-1). Patients' quality of life level was evaluated using inhouse self-made comprehensive quality of life assessment questionnaire, including indicators such as psychological function, body function and life function. In addition, a simple FuglMeyer assessment (FMA) exercise ability evaluation method was adopted to evaluate the neurological function. Statistical analysis software SPSS21.0 was used to process data. The measurement data were expressed by mean \pm standard deviation (SD), with t-test for intergroup comparison. Enumeration data was expressed by natural (n) numbers and percentage (\%), with $\mathrm{X}^{2}$ used for intergroup comparison. The intergroup difference is of statistical value when $P<0.05$

According to results presented in Table 1 improvement in all indices after treatment in the research group was significantly better than that in the control group, $(P<0.05)$. As shown in Table 2, the levels of immune markers of inflammation after treatment were observed. The results showed that serum TNF- $\alpha$, IL- 6 and ICAM1 concentrations were significantly reduced in the research group, $(P<0.05)$. No significant differences were found between these two groups for other indexes. As shown in Table 3, the research group had significant advantages in all index scores $(P<0.05)$. As shown in Table 4, compared to the control group, the FMA exercise ability score of the research group showed significant advantage $(P<0.05)$. The comparison of NIHSS scores before and after treatment is shown in fig. 3 .

Many studies have shown ${ }^{[13,14]}$ that atorvastatin has direct antiinflammatory effects in addition to lipidlowering effect. Atorvastatin can prevent the rupture of atherosclerotic plaques, reduce the number of macrophages in atherosclerotic plaques, inhibit the expression of matrix proteases, prevent the destruction of the plaque fibrocap and prevent monocytes from adhering to the endothelial cell wall. Clinically, large retrospective studies, such as air force/Texas coronary atherosclerosis prevention study, pravastatin inflammation/CRP evaluation (PRINCE) $)^{[15]}$ have confirmed the significant antiinflammatory effect of atorvastatin and its ability to reduce hsCRP after longterm treatment. Some studies pointed out ${ }^{[16]}$ that the level of dhsCRP was significantly reduced 7-14 d after atorvastatin treatment. This shows that atorvastatin could effectively reduce hsCRP and has potential application value in antiinflammatory therapy for patients with acute cerebral infarction. Therefore, it is reasonable and necessary to include atorvastatin in the treatment of acute cerebral infarction. 
TABLE 1: BLOOD LIPID PROFILE OF THE TWO GROUPS BEFORE AND AFTER TREATMENT

\begin{tabular}{lcccccccc}
\hline \multirow{2}{*}{ Group } & \multicolumn{2}{c}{ TC $(\mathrm{mM})$} & \multicolumn{2}{c}{ TG $(\mathrm{mM})$} & \multicolumn{2}{c}{ LDL-C $(\mathrm{mM})$} & \multicolumn{2}{c}{ HDL-C $(\mathrm{mM})$} \\
\cline { 2 - 8 } & Before & After & Before & After & Before & After & Before & After \\
\hline Research & $5.29 \pm 1.20$ & $2.18 \pm 0.73$ & $1.88 \pm 0.65$ & $0.96 \pm 0.21$ & $3.74 \pm 1.18$ & $1.42 \pm 0.50$ & $1.36 \pm 0.35$ & $0.69 \pm 0.15$ \\
Control & $5.48 \pm 1.17$ & $3.69 \pm 0.99$ & $1.83 \pm 0.61$ & $1.56 \pm 0.52$ & $3.85 \pm 1.20$ & $2.26 \pm 0.55$ & $1.39 \pm 0.42$ & $0.98 \pm 0.28$ \\
$\mathrm{t}$ & 0.28 & 9.36 & 0.11 & 9.04 & 0.22 & 8.37 & 0.13 & 6.70 \\
$P$ & $>0.05$ & $<0.05$ & $>0.05$ & $<0.05$ & $>0.05$ & $<0.05$ & $>0.05$ & $<0.05$ \\
\hline
\end{tabular}

TABLE 2: IMMUNE INFLAMMATORY MARKERS OF THE TWO GROUPS

\begin{tabular}{lccccccccc}
\hline Group & $\begin{array}{c}\text { CRP } \\
(\mathbf{m g} / \mathrm{dl})\end{array}$ & $\begin{array}{c}\text { TNF-a }(\mathrm{pg} / \\
\mathrm{dl})\end{array}$ & $\begin{array}{c}\mathrm{IL}-1 \\
(\mathbf{p g} / \mathrm{dl})\end{array}$ & $\begin{array}{c}\mathrm{IL}-6 \\
(\mathbf{p g} / \mathrm{dl})\end{array}$ & $\begin{array}{c}\mathrm{IL}-10 \\
(\mathrm{pg} / \mathrm{dl})\end{array}$ & $\begin{array}{c}\text { E-selectin } \\
(\mathrm{pg} / \mathrm{dl})\end{array}$ & $\begin{array}{c}\text { P-selectin } \\
(\mathbf{p g} / \mathrm{dl})\end{array}$ & $\begin{array}{c}\mathrm{VCAM}-1 \\
(\mathrm{pg} / \mathrm{dl})\end{array}$ & $\begin{array}{c}\text { ICAM-1 } \\
(\mathbf{p g} / \mathrm{dl})\end{array}$ \\
\hline Research & $2.4 \pm 0.6$ & $16.0 \pm 1.9$ & $8.7 \pm 0.6$ & $8.6 \pm 1.3$ & $3.8 \pm 0.94$ & $2.5 \pm 0.5$ & $3.8 \pm 0.7$ & $17 \pm 2.1$ & $13.6 \pm 2.1$ \\
Control & $2.7 \pm 0.9$ & $22.5 \pm 1.8$ & $9.6 \pm 1.2$ & $10.6 \pm 1.2$ & $3.9 \pm 0.8$ & $2.5 \pm 0.3$ & $3.6 \pm 0.9$ & $18.9 \pm 3.2$ & $18.6 \pm 3.0$ \\
$\mathrm{t}$ & & 9.03 & 1.05 & 12.16 & 0.21 & 0.47 & 1.02 & 0.23 & 8.90 \\
$P$ & $>0.05$ & $<0.05$ & $>0.05$ & $<0.05$ & $>0.05$ & $>0.05$ & $>0.05$ & $>0.05$ & $<0.05$ \\
\hline
\end{tabular}

TABLE 3: COMPARISON OF LIFE QUALITY SCORE OF THE TWO GROUPS

\begin{tabular}{lccccc}
\hline Group & Number of cases & Time & Body function & Mental function & Living function \\
\hline Research & 90 & 3 d after admission & $13.2 \pm 0.63$ & $14.6 \pm 0.69$ & $14.0 \pm 0.52$ \\
& & 1 mo after admission & $23.4 \pm 0.75$ & $20.6 \pm 0.73$ & $21.6 \pm 0.78$ \\
Control & 90 & 3 d after admission & $13.7 \pm 0.64$ & $14.2 \pm 0.75$ & $14.1 \pm 0.82$ \\
& & 1 mo after admission & $16.9 \pm 0.53$ & $17.6 \pm 0.48$ & $18.5 \pm 0.91$ \\
\hline
\end{tabular}

TABLE 4: FMA EXERCISE ABILITY SCORES OF THE TWO GROUPS

\begin{tabular}{lccc}
\hline Group & $\begin{array}{c}\text { Number of } \\
\text { cases }\end{array}$ & $\begin{array}{c}3 \text { d after } \\
\text { admission }\end{array}$ & $\begin{array}{c}1 \text { mo after } \\
\text { admission }\end{array}$ \\
\hline Research group & 90 & $52.7 \pm 0.96$ & $74.6 \pm 1.40$ \\
Control group & 90 & $51.9 \pm 0.86$ & $66.3 \pm 1.35$ \\
$T$ & & 0.18 & 4.37 \\
$P$ & $>0.05$ & $<0.05$ \\
\hline
\end{tabular}

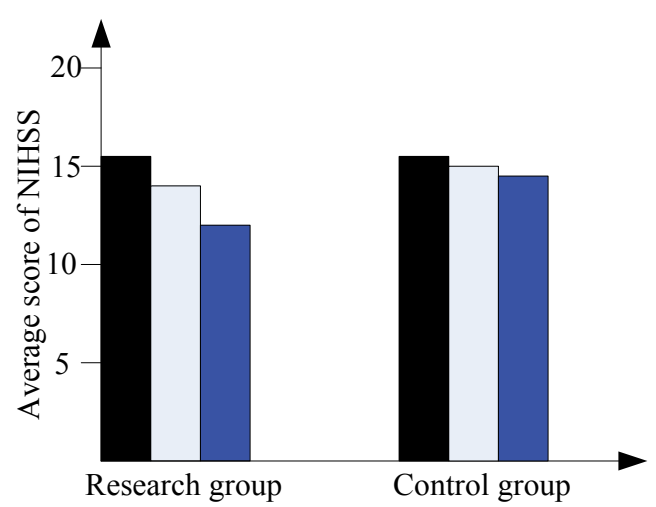

Fig. 3: Comparison of NIHSS scores before and after treatment

(ロ) Before treatment, ( $\square$ ) 3 days after treatment ( $\square$ ) 7 days after treatment

The contents of comprehensive nursing intervention can be described by the following 5 points. First, psychological nursing. The nurses should conduct positive communication with patients to have a certain understanding of their current psychological status and apply targeted and individualized programs to help them channel bad emotions. Second, conduct health promotion education to strengthen belief. Doctors should teach patients the causes, treatment approaches, medication status, treatment effect and precautions to be taken after acute cerebral infarction so as to enable the patients to have correct understanding of the disease, strictly follow medical advice for good treatment compliance and be able to consciously carry out selfmanagement. In addition, education is also carried out for the family members of patients to encourage them to have a positive and optimistic attitude when caring for the patients and to spread positive energy to the patients. Third, peer effects. The department should organize the group study of patients in the department's teaching and research office every week, so that everyone can gain more useful treatment experience, improve the communication between patients, encourage each other, and jointly fight against the disease. Fourthly, the patient is provided with a quiet, comfortable, clean and neat hospital environment with bedsheets frequently changed, so that the patient is well cared and the patient is provided with a quiet sleeping environment at night. Fifth, develop a healthy diet plan for patients, avoiding spicy food. Sixth, the guidance on medication method. Pay attention to the time, dosage and precautions of each medication and maintain a detailed record of patients' medication situation, so that patients can timely check and improve medical compliance.

In conclusion, the antiinflammatory effect of atorvastatin may overlap with the dynamic changes 
of inflammatory markers in acute cerebral infarction. Previous studies using platelet activation markers in patients with stable or unstable cardiovascular disease have shown that statins have antiplatelet effects, which is distinct from the effect of lowering cholesterol. Other studies have shown that atorvastatin can inhibit oxidative stress and platelet activation by directly inhibiting platelet NOX2/platelet prostaglandin and thrombin A2, which provides a theoretical basis for statin drug inhibition or regulation of thrombosis. Many studies have shown that atorvastatin has direct antiinflammatory effects in addition to the serum cholesterol lowering effect. In addition, with scientific nursing intervention measures, the effect of adjuvant treatment can be brought into full play. Therefore, using atorvastatin to treat patients with acute cerebral infarction can produce good antiinflammatory effects, and the adoption of comprehensive nursing measures can enable patients to achieve a higher quality of life. Therefore, this treatment is worth of being promoted in clinics.

\section{Acknowledgments:}

Health-promoting Effect of "The Constitutional Foodadjusting Theory" on Sub-healthy Group (Primary Health Development Research Center of Sichuan Province: SWFZ17-Y-45).

\section{REFERENCES}

1. Zhou Y, Wang WX, Pan TF, Meng LH, Wang H. Effects of atorvastatin on blood lipids, fibrinogen and hs-CRP in patients with acute cerebral infarction. Mod J Integr Tradi Chinese West Med 2017; 26(16):1792-4.

2. Chen Q, Zheng LC, Yang Z. Effect of atorvastatin on serum hypersensitive c-reactive protein interleukin-17 and matrix metalloproteinase- 8 in patients with acute cerebral infarction. Chinese J Prac Nerv Dis 2015;18(03):9-11.

3. Mou DH. Analysis the efficacy in treatment of acute cerebral infarction with reinforcement amount of atorvastatin calcium. World Latest Med Info 2016;16(12):70-1.

4. Iwata H, Masuda N, Ohno S, Rai Y, Sato Y, Ohsumi S, et al. A randomized, double-blind, controlled study of exemestane versus anastrozole for the first-line treatment of postmenopausal Japanese women with hormone-receptor-positive advanced breast cancer. Breast Cancer Res Treat 2016; 139(2):441-51.

5. Lee DS, Kim SH, Kim S, Suh YJ, Kim HK, Shim BY. Prognostic significance of breast cancer subtype and p53 overexpression in patients with locally advanced or high-risk breast cancer treated using upfront modified radical mastectomy with or without post-mastectomy radiation therapy. Int J Clin Oncol 2017;17(5):447-55.
6. Zhang Li, Huang Jing, Li Meng. Effects of probucol combined with atorvastatin on serologic indexes in convalescent patients with acute cerebral infarction. J Hainan Med Univ 2016; 22(11):1160-3.

7. Saghir A, Nafees B, Jehan B, Jawad A. Characterization of pathogens involved in ventilator associated pneumonia in surgical and medical intensive care units - a single center experience. Pak J Pharm Sci 2017;30(6):2091-9.

8. AlQuadeib BT, Eltahir EKD, RA Banafa, Al-Hadhairi LA. Pharmaceutical evaluation of different shampoo brands in local saudi market. Saudi Pharm J 2018; 26(1):98-106.

9. Demirağ F, Yilmaz A, Yilmaz DN, Yilmaz Ü, Erdoğan Y. Egfr, kras, and braf mutational profiles of female patients with micropapillary predominant invasive lung adenocarcinoma. Turk J Med Sci 2017; 47(5):1354-61.

10. Khajuria DK, Razdan R. Sensitive and rapid rp-hplc quantification of zoledronic acid in a hydroxyapatite-based nanoparticles. Indian J Pharm Sci 2017;79(4): 553-8.

11. Lo Sasso B, Agnello L, Florena AM, Pappalardo E, Milano $\mathrm{S}$, Butera $\mathrm{D}$, et al. The cervical fracture as first symptom of multiple myeloma: a case report. Acta Medica Mediterr 2017;33(3): 535-8.

12. Wanxi P, Zhi L, Lansheng W, Junbo C, Fangliang GU, Xiangwei Z. Molecular characteristics of illicium verum extractives to activate acquired immune response. Saudi J Biol Sci 2016;23(3): 348-52.

13. Cheng GH, Li WD, Qian JN. Effect of different doses of atorvastatin calcium on acute cerebral infarction. China Mod Med 2017;24(33):88-90.

14. Cantey JB, Sreeramoju P, Jalee M. Prompt control of an outbreak caused by extended-spectrum $\beta$-lactamase-producing klebsiella pneumoniae in a neonatal intensive care unit. J Pediatr 2017;163(3):672-9.

15. Badal RE, Bouchillon SK, Lob SH. Etiology, extendedspectrum $\beta$-lactamase rates and antimicrobial susceptibility of gram-negative bacilli causing intra-abdominal infections in patients in general pediatric and pediatric intensive care units - Global data from the study for monitoring antimicrobial resistance trends 2008 to 2010. Pediatr. Infect Dis J 2016; 32(6):636-40.

16. Markovska RD, Stoeva TJ, Bojkova KD. Epidemiology and molecular characterization of extended-spectrum betalactamase-producing enterobacter spp., pantoeaagglomerans, and Serratia marcescens isolates from a Bulgarian hospital. Microb Drug Resist 2017; 20(2):131-7.

This is an open access article distributed under the terms of the Creative Commons Attribution-NonCommercial-ShareAlike 3.0 License, which allows others to remix, tweak, and build upon the work non-commercially, as long as the author is credited and the new creations are licensed under the identical terms

This article was originally published in a special issue: Special issue on "Animal Models \& Experimental Medicine"

Indian J Pharm Sci 2020:82(1)spl issue4;67-71 